TRABAJOS ORIGINALES

\title{
Uso de hábitat y estado de conservación de las aves en el humedal EI Paraíso, Lima, Perú
}

\author{
Habitat use and conservation status of birds from El Paraíso wetland, Lima, Peru
}

\author{
Alessandra S. Quiñonez ${ }^{1,2}$, Flor Hernandez ${ }^{1}$ \\ 1 Centro de Ornitología y Biodiversidad. CORBIDI. Calle Sta Rita 105 of 202. Huertos de San Antonio, Surco, Lima, Perú \\ 2 Universidad Peruana Cayetano Heredia. Calle Honorio Delgado 430, S.M.P, Lima, Perú. \\ Email Alessandra S. Quiñonez: aquinonez@corbidi.org \\ Email Flor Hernandez: fhernandez@corbidi.org \\ ORCID Alessandra Quiñonez: 0000-0002-2165-9985
}

\begin{abstract}
Resumen
Las aves son el grupo taxonómico más representativo de los humedales. En la costa central peruana muchos de estos ecosistemas se encuentran seriamente afectados por actividades antropogénicas. Con el objetivo de brindar información que contribuya a la implementación de planes de manejo y conservación de la avifauna del humedal El Paraíso se evaluó las preferencias y usos de los dos hábitats acuáticos presentes en el humedal, la laguna salobre y la zona cenagosa. Se llevaron a cabo evaluaciones mensuales desde mayo del 2009 a marzo del 2010 utilizando el método de puntos de conteo. Se observaron 68 especies que hicieron uso directo de los hábitats de estudio. Las familias más abundantes fueron Anatidae (19.2\%), Laridae (18.0\%), Phalacrocoracidae (16.8\%) y Rallidae (16.1\%). La especie más abundante fue Phalacrocorax brasilianus (16.7\%), seguida de la Fulica ardesiaca (13.0\%). Se encontró marcada diferencias en la composición de aves entre los dos hábitats evaluados debido, principalmente, a la presencia de especies migratorias boreales quienes prefieren el hábitat cenagoso. Por otro lado, fueron identificados los sitios de anidamiento de especies representativas del humedal tales como Phalacrocorax brasilianus, Tachuris rubrigastra, Phleocryptes melanops, Charadrius nivosus. La actividad frecuentemente registrada fue el descanso (73.9\%), seguido de la alimentación (23.4\%) y la reproducción (2.73\%). Constituyen las principales amenazas para el humedal; la caza deportiva, quema de totorales y juncales, tránsito de vehículos por la orilla marina y desechos de residuos sólidos dejados por los bañistas.
\end{abstract}

Palabras clave: El Paraíso; humedal; aves; uso de hábitat; conservación.

\section{Abstract}

Birds are the most representative group of wetlands. In the Central Peruvian coast many of these ecosystems are severely affected by anthropogenic activities, for instance, El Paraíso wetland. The aim of this study is to evaluate the preferences and habitat uses of two aquatic bodies: brackish lagoon and marshy area at El Paraíso Wetland, in order to gather information for the implementation of management plans and conservation of this wetland. From May 2009 to March 2010, we carried out monthly assessment by point counts technique. We identified 68 species that made direct use of the studied habitats. The most abundant families were Anatidae (19.2\%), Laridae (18.0\%), Phalacrocoracidae $(16.8 \%)$ and Rallidae (16.1\%). The most abundant species was Phalacrocorax brasilianus (16.7\%), followed by the Fulica ardesiaca (13.0\%). We found marked differences in the composition of birds' communities between the two evaluated habitats, which mainly occurred due to the presence of boreal migratory species that prefer swampy habitat. Nesting sites of representative species of wetland such as, Phalacrocorax brasilianus, Tachuris rubrigastra, Phleocryptes melanops and Charadrius nivosus were recorded. The most common bird activity recorded was resting $(73.9 \%)$, followed by feeding $(23.4 \%)$ and breeding $(2.73 \%)$. The main threats to the wetland are, hunting, burning (cattails and rushes), vehicular traffic by seashore, solid waste and debris left by the swimmers.

Keywords: El Paraíso; wetland; birds; habitat use; conservation.

Citación:

Quiñonez A.S., F. Hernandez. 2017. Uso de hábitat y estado de conservación de las aves en el humedal El Paraíso, Lima, Perú. Revista peruana de biología 24(2): 175 - 186 (Julio 2017). doi: http://dx.doi. org/10.15381/rpb.v24i2.13494

$\begin{array}{ll}\text { Presentado: } & 18 / 03 / 2016 \\ \text { Aceptado: } & 20 / 01 / 2017\end{array}$

Publicado online: $20 / 07 / 2017$
Información sobre los autores:

ASQ y FH realizaron el diseño, trabajo de campo, analisis de datos: redactaron y aprobaron el manuscrito.

Los autores no incurren en conflictos de intereses. 


\section{Introducción}

En la conservación y uso sostenible de los humedales, la información sobre el uso del hábitat por parte de las aves es un elemento importante para la planificación, manejo territorial y conservación, debido a que constituyen uno de los componentes más característicos de la fauna que habita en estos ecosistemas (Myers 1983, Weller 1999, Blanco 1999, Angulo 2009).

Los humedales ofrecen a las aves: refugio, alimento y áreas de reproducción (Blanco 1999, Tabilo 2003). Además son áreas de vital importancia para especies migratorias (Nuñez 1999, Torres et al. 2006).

La distribución y uso del hábitat por parte de las aves está asociado a características ambientales locales y a requerimientos particulares de cada especie (Blanco 1999, Torres 2007, Weller 1999). Una de las variables importantes en la distribución de las aves se relaciona con la disponibilidad y concentración temporal del alimento (Weller 1999, Chudzińska et al. 2015). Así mismo el hábitat, a nivel de paisaje, es otro de los factores ambientales que define el patrón de diversidad y el tipo de comunidad (Weller 1999, Brandolin \& Blendinger 2016). El humedal el Paraíso, es considerado uno de los humedales más importantes y estratégicos de la costa peruana dada su alta diversidad y variedad de hábitats (DS N004-2015-MINAN). Además ha sido reconocida como un Área Importante para la Conservación de las Aves, IBA PE030 (BirdLife 2016).

El clima de El Paraíso es desértico, cálido y húmedo. Forma parte de la eco-región del complejo Desértico Pacífico Costero (Brack 1986, MINAM 2011). La vegetación en el humedal es típica de lagunas similares en la costa (Cruz et al. 2007) y la fauna está representada principalmente por las aves.

Los diferentes estudios sobre la avifauna del Paraíso, han registrado entre 81 y 109 especies (Riveros et al. 1983; Castro 1984; Castro et al. 1990; Velarde, 1998; Cruz et al. 2007, Tello et al. 2008).

Con el objetivo de brindar información que contribuya a la implementación de planes de manejo y conservación de la avifauna de El Paraíso se evaluó las preferencias y usos de los hábitats acuáticos, laguna salobre y zona cenagosa. Además se identificó espacialmente las actividades antropogénicas que estarían afectando negativamente a la comunidad de aves de El Paraíso.

\section{Materiales y métodos}

Área de estudio.- La laguna El Paraíso se ubica en el distrito de Huacho, provincia de Huaura, Región Lima, aproximadamente a $136 \mathrm{~km}$ al norte de la ciudad de Lima y a $10 \mathrm{~km}$ al sur

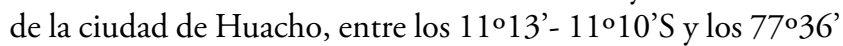
- 77035’W. Su superficie actual es de 475.3 ha aproximadamente (Cruz et al. 2007) y tiene una longitud de 7 kilómetros, con un ancho que varía de 0.1 a 1 kilometro (Riveros et al. 1983, Castro et al. 1990) (Fig. 1).

El humedal está compuesto por tres hábitats acuáticos, laguna salobre, laguna pantanosa y mar abierto (Scout \& Carbonel 1986). En este estudio sólo se evaluaron los hábitats de laguna salobre y laguna cenagosa (pantanosa) cuyo régimen hidrológico se encuentra influenciado por elevaciones de la napa freática,

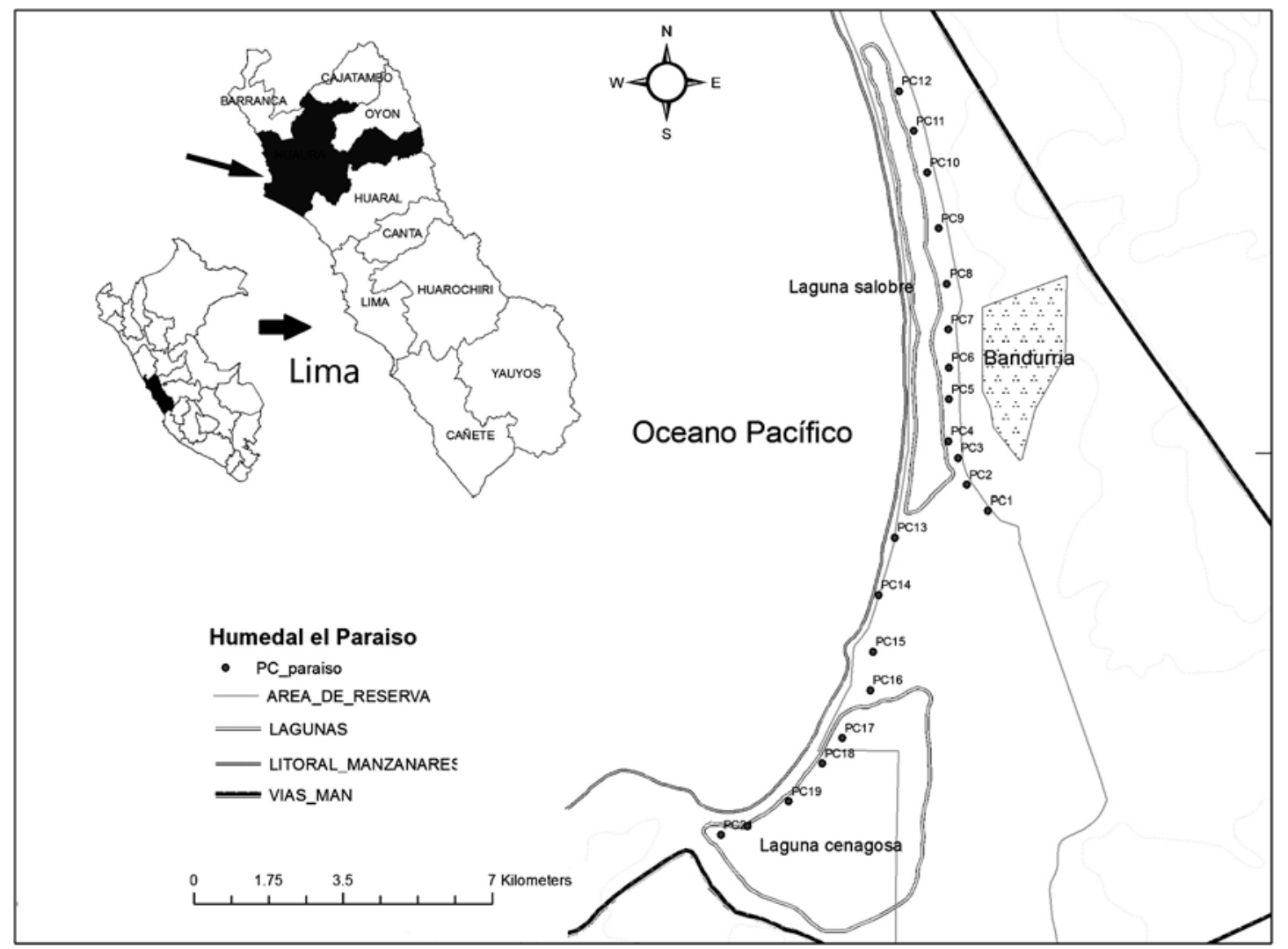

Figura 1. Humedal el Paraiso, Huacho, Perú. Se grafican los puntos de conteo (PC) empleados en el presente estudio. Elaboración propia. 
las mareas y por el agua proveniente de la irrigación Santa Rosa (BirdLife International 2016, Tello et al. 2008).

La laguna salobre tiene un área aproximada de 58 ha, es de forma alargada, registra como profundidad máxima $150 \mathrm{~cm}$, la conductividad eléctrica promedio es de $8.66 \mathrm{mS}$ y el $\mathrm{pH}$ es de 9.39 (Riveros et al. 1983, Castro et al. 1990, Tello Rodríguez et al. 2014). La vegetación predominante alrededor de la laguna está compuesta por: totorales, juncales y gramadales.
La laguna cenagosa, tiene un área aproximada de 195 ha, contiene más sales que la laguna salobre registrando una conductividad promedio de $15.7 \mathrm{mS}$, que puede llegar a $20.3 \mathrm{mS}$ en la zona de influencia de las mareas. Presenta $\mathrm{pH}$ alcalino de 8.65 (Riveros et al. 1983, Castro et al. 1990). La vegetación predominante en esta área son vega de ciperáceas, salicornial y juncales. La descripción de la vegetación en cada punto de evaluación es mostrada en la Tabla 1.

Tabla 1. Caracterización de la vegetación aledaña a cada punto de conteo PC.

\begin{tabular}{|c|c|c|c|}
\hline $\begin{array}{l}\text { Punto de } \\
\text { conteo }\end{array}$ & Latitud & Longitud & Comunidad Vegetal \\
\hline PC 1 & $11^{\circ} 11.708^{\prime}$ & $77^{\circ} 35.247^{\prime}$ & $\begin{array}{l}\text { Carrizal / Gramadal con cf. Arundo donax y Paspalum vaginatum como especies dominantes. Otras } \\
\text { especies presentes: Tessaria integrifolia y Scirpus americanus. }\end{array}$ \\
\hline PC 2 & $11^{\circ} 11.605^{\prime}$ & $77^{\circ} 35.332^{\prime}$ & $\begin{array}{l}\text { Gramadal con Paspalum vaginatum y Scirpus americanus como especies dominantes. Otras especies } \\
\text { presentes: Cynodon dactylon y Schoenoplectus californicus. }\end{array}$ \\
\hline PC 3 & $11^{\circ} 11.498^{\prime}$ & $77^{\circ} 35.365^{\prime}$ & $\begin{array}{l}\text { Gramadal con Distichlis spicata como especies dominante. Totoral con Schoenoplectus californicus y } \\
\text { Typha domingensis como especies dominantes. Otras especies presentes: cf. Arundo donax. }\end{array}$ \\
\hline PC 4 & $11^{\circ} 11.431$ & $77^{\circ} 35.404$ & $\begin{array}{l}\text { Porcion de laguna con Schoenoplectus californicus como especie dominante en el borde este y Typha } \\
\text { domingensis en el borde oeste (totoral). }\end{array}$ \\
\hline PC 5 & $11^{\circ} 11.264^{\prime}$ & $77^{\circ} 35.400^{\prime}$ & $\begin{array}{l}\text { Porcion de laguna con S. americanus como especie dominante en el borde este con Sesuvium } \\
\text { portulacastrum y Sarcocornia fruticosa. Borde oeste con Typha domingensis como especie dominante } \\
\text { (totoral). }\end{array}$ \\
\hline PC 6 & $11^{\circ} 11.138^{\prime}$ & $77^{\circ} 35.400^{\prime}$ & $\begin{array}{l}\text { Porcion de laguna con S. americanus y S. californicus como especies dominantes en el borde este } \\
\text { con pequeños parches de Sesuvium portulacastrum y Sarcocornia fruticosa. Borde oeste con Typha } \\
\text { domingensis como especie dominante. }\end{array}$ \\
\hline PC 7 & $11^{\circ} 10.986^{\prime}$ & $77^{\circ} 35.400^{\prime}$ & $\begin{array}{l}\text { Porcion de laguna con gramadal en el borde este con Paspalum vaginatum y Distichlis spicata como } \\
\text { especies dominantes. Otras especies presentes en el borde este: Cyperus laevigatus y S. californicus, } \\
\text { Scirpus maritimus, S. americanus y S. fruticosa. Typha domingensis dominante en lado oeste (totoral). }\end{array}$ \\
\hline PC 8 & $11^{\circ} 10.804^{\prime}$ & $77^{\circ} 35.404$ & $\begin{array}{l}\text { Porcion de laguna con S. americanus y C. laevigatus como especies dominantes en el borde este con } \\
\text { presencia de S. portulacastrum. Borde oeste dominado por T. domingensis. }\end{array}$ \\
\hline PC 9 & $11^{\circ} 10.582^{\prime}$ & $75^{\circ} 35.436$ & $\begin{array}{l}\text { Porcion de laguna con S. americanus y D. spicata como especies dominantes en el borde este con } \\
\text { presencia de S. portulacastrum y S. fruticosa. Borde oeste dominado por T. domingensis. }\end{array}$ \\
\hline PC 10 & $11^{\circ} 10.360$ & $77^{\circ} 35.480$ & $\begin{array}{l}\text { Porcion de laguna con } S \text {. americanus como especie dominante en el borde este con presencia de } D \text {. } \\
\text { spicata; S. fruticosa y T. domingensis. Borde oeste dominado por T. domingensis. }\end{array}$ \\
\hline PC 11 & $11^{\circ} 10.194^{\prime}$ & $77^{\circ} 35.533$ & $\begin{array}{l}\text { Porcion de laguna con } S \text {. americanus y D. spicata como especies dominantes en el borde este con } \\
\text { presencia de T. dominguensis, S. californicus y S. fruticosa. Borde oeste dominado por T. domingensis. }\end{array}$ \\
\hline PC 13 & $11^{\circ} 11.811^{\prime}$ & $77^{\circ} 35.624^{\prime}$ & $\begin{array}{l}\text { Totoral / Gramadal / Juncal. Especies dominantes: D. spicata, S. americanus y T. domingensis. Otras } \\
\text { especies presentes: S. fruticosa. }\end{array}$ \\
\hline PC 14 & $11^{\circ} 12.039^{\prime}$ & $77^{\circ} 35.692$ & $\begin{array}{l}\text { Totoral / Gramadal / Juncal. Especies dominantes: D. spicata, S. americanus y T. domingensis. Otras } \\
\text { especies presentes: S. fruticosa. }\end{array}$ \\
\hline PC 15 & $11^{\circ} 12.265^{\prime}$ & $77^{\circ} 35.715^{\prime}$ & $\begin{array}{l}\text { Cuerpo de agua dominado en los bordes por } S \text {. americanus, por } S \text {. fruticosa hacia el oeste y } S \text {. } \\
\text { portulacastrum cerca al arenal }\end{array}$ \\
\hline PC 16 & $11^{\circ} 12.418^{\prime}$ & $77^{\circ} 35.728^{\prime}$ & $\begin{array}{l}\text { Cuerpo de agua dominado en los bordes por } S \text {. maritimus, por P. vaginatum hacia el oeste y } S \text {. } \\
\text { fruticosa cerca al arenal. }\end{array}$ \\
\hline PC 17 & $11^{\circ} 12.608$ & $77^{\circ} 35.843^{\prime}$ & $\begin{array}{l}\text { Cuerpo de agua dominado en los bordes por S. maritimus (Juncal o Vega de Ciperaceas) y por } S \text {. } \\
\text { fruticosa hacia el oeste (salicornial). }\end{array}$ \\
\hline PC 18 & $11^{\circ} 12.708^{\prime}$ & $77^{\circ} 35.925$ & $\begin{array}{l}\text { Cuerpo de agua dominada en los bordes por S. maritimus (Juncal o Vega de Ciperaceas) y por } S \text {. } \\
\text { fruticosa hacia el oeste (salicornial). }\end{array}$ \\
\hline PC 19 & $11^{\circ} 12.858^{\prime}$ & $77^{\circ} 36.060$ & $\begin{array}{l}\text { Cuerpo de agua dominada en los bordes por S. maritimus (Juncal o Vega de Ciperaceas) y por } S \text {. } \\
\text { fruticosa hacia el oeste (salicornial). }\end{array}$ \\
\hline PC 20 & $11^{\circ} 12.956^{\prime}$ & $77^{\circ} 36.227^{\prime}$ & $\begin{array}{l}\text { Cuerpo de agua dominado en los bordes por S. maritimus (Juncal o Vega de Ciperaceas) y por } S \text {. } \\
\text { fruticosa hacia el oeste (salicornial). }\end{array}$ \\
\hline PC 21 & $11^{\circ} 12.989^{\prime}$ & $77^{\circ} 36.336^{\prime}$ & Conexión Laguna / Mar dominado por S. fruticosa (Salicornial) al margen derecho. \\
\hline
\end{tabular}




\section{Materiales y métodos}

Se llevaron a cabo evaluaciones mensuales de mayo del 2009 a marzo del 2010. Cada evaluación tuvo una duración de dos días cubriendo la mańana $(8: 00$ - 13:00 h) y la tarde (14:00 - 17:00 h). El primer día se iniciaba por la zona norte y terminaba en la zona sur del humedal, y el segundo día se iniciaba de forma inversa.

Se utilizó el método de puntos de conteo fijo en un transecto (Bibby et al. 2000). Para dicha metodología se dividió espacialmente la laguna en dos transectos: norte y sur. Se establecieron $12 \mathrm{PC}$ en el transecto norte y $9 \mathrm{PC}$ en el transecto sur (Fig. 1). Cada PC tuvo una duración de $10 \mathrm{~min}$, y la distancia entre los puntos fue de $200 \mathrm{~m}$ aproximadamente. Se utilizó este método de evaluación debido a que da la ventaja de evaluar con detenimiento una amplia variedad de aves y su comportamiento.

La información colectada fue: fecha, PC, zona, tipo de hábitat, hora inicio y fin, especie, sexo, categoría de edad (si fuera el caso) y comportamiento observado. Este último ítem se clasificó según las siguientes categorías:

Alimentación, forrajeo, captura e ingesta de alimentos.

Descanso, incluye las actividades de nadar, acicalarse, percha, interacciones como el juego y la agresión.

Reproducción, incluye cuidado parental, nidificación, cortejo y cópula.

Cada censo contó con un observador y un anotador, quienes utilizaron binoculares Eagle Optics ${ }^{\circledR} 8 \times 42$, además de una cámara fotográfica Colpix p100 Nikon ${ }^{\circledR}$. Para corroborar la identificación taxonómica se utilizó la guía de campo Birds of Perú (Shulenberg et al. 2010).

El uso de hábitat fue analizado en función a la abundancia de especies de aves avistadas en la laguna salobre y laguna cenagosa, considerado a aquellas que se registraron de forma única en alguno de los dos hábitats y a aquellas que se encontraban en los dos hábitats de forma indistinta. Las especies registradas por única vez no fueron incluidas en el análisis, pero fueron consideradas en la información de riqueza.

Con el objetivo de evaluar la variación espacial y temporal de la comunidad de aves se realizó un análisis multivariado empleando la metodología de clúster. Este tipo de análisis permite comparar simultáneamente una serie de muestras e ilustrar las relaciones existentes entre ellas. Para efectos del presente estudio se utilizó el índice de similitud de Raup-Crick el cual toma en cuenta la ausencia y la presencia de las especies en cada uno de los puntos de conteo. Los análisis se realizaron mensualmente.

Evaluación de variables fisicoquímicas del agua.- En junio y octubre del 2009, se tomaron muestreos de agua en cada una de las lagunas con el objetivo de caracterizarlas. Las variables medidas fueron: profundidad, temperatura, $\mathrm{pH}$ y oxígeno disuelto (Tabla 2).

El muestreo de $\mathrm{pH}$ y oxígeno disuelto se realizó con un equipo colorimétrico proporcionado por la gerencia de Recursos Naturales y Recursos de medio ambiente del Gobierno Regional de Lima.

La profundidad (nivel de agua) fue tomada utilizando una vara de madera graduada $(\mathrm{cm})$, y de forma simultánea niveles de agua indicados por los hidrómetros presentes al borde de la laguna norte colocados por el Proyecto de conservación y manejo sostenible de los humedales de la costa central. Las muestras fueron tomadas a los bordes de cada laguna.

Todos los análisis estadísticos se realizaron con el programa PAST (Hammer et al. 2001).

Registro de actividades antropogénicas.- Se registraron todas las actividades antropogénicas realizadas dentro del humedal. Las actividades que generan una afectación negativa en la comunidad de aves fueron identificadas en un mapa. Así mismo se mapearon las zonas de mayor vulnerabilidad en función a actividades claves como: la reproducción y alimentación de especies.

\section{Resultados y discusión}

Composición de especies.- Durante la evaluación se identificaron 68 especies que hicieron uso directo de los hábitats de estudio (Tabla 3). Las familias más abundantes fueron: Anatidae (19.2\%), Laridae (18.0\%), Phalacrocoridae (16.8\%) y Rallidae (16.1\%). La especie más abundante fue Phalacrocorax brasilianus (16.7\%), seguida de la Fulica ardesiaca (13.0\%).

Algunas las de las especies registradas se encuentran dentro de las listas de especies amenazadas. Tal es el caso de Pelecanus thagus, Sternula lorata, categorizados como En Peligro (IUCN), y Phoenicopterus chilensis, categorizada como Casi Amenazada (IUCN).

De todas las especies registradas, algunas fueron raras (de registros ocasionales o poco comunes para el humedal. Tal es el caso del Anas geórgica, Egretta tricolor, Gelochelidon nilotica, Falco peregrinus, y Asio flammeus. Además, durante el estudio se registró una nueva especie para el área, Coccyzus melacoryphus (Quiñonez \& Tello 2011).

Tabla 2. Parámetros fisicoquímicos registradas en junio y octubre del año 2009.

\begin{tabular}{|c|c|c|c|c|c|c|c|}
\hline \multirow[b]{2}{*}{ Mes } & \multirow[b]{2}{*}{ Variable } & \multicolumn{3}{|c|}{ Laguna Salobre } & \multicolumn{3}{|c|}{ Laguna Cenagosa } \\
\hline & & 1 & 2 & 3 & 1 & 2 & 3 \\
\hline \multirow[t]{4}{*}{ Junio } & Profundidad $(\mathrm{cm})$ & 90.5 & 68 & 78 & 20 & 19.5 & 12.5 \\
\hline & Temperatura $\left(\mathrm{C}^{\circ}\right)$ & 20.3 & 21.2 & 22.1 & 21.2 & 19.5 & 19 \\
\hline & Oxígeno disuelto & 9.3 & 9.2 & 9.2 & 16.6 & 10.2 & 10.2 \\
\hline & $\mathrm{pH}$ & 7.6 & 8.1 & 8.4 & 7.4 & 7.6 & 7.4 \\
\hline \multirow[t]{4}{*}{ Octubre } & Profundidad (cm) & 37 & 33 & 32.5 & 2.8 & 2.6 & 6.6 \\
\hline & Temperatura $\left(\mathrm{C}^{\circ}\right)$ & 18.5 & 19.5 & 21 & 24.5 & 27 & 24.5 \\
\hline & Oxígeno disuelto & 3.8 & 3 & 6 & 8.9 & 12.8 & 13.6 \\
\hline & $\mathrm{pH}$ & 7 & 7 & 7.5 & 7.7 & 8 & 8.1 \\
\hline
\end{tabular}


Preferencia y uso de hábitat.- La preferencia de hábitats por parte de las aves en el humedal El Paraíso se distribuye principalmente en los dos hábitats acuáticos evaluados. Se evidenció un patrón de agrupación entre los $\mathrm{PC}$ relacionados a la laguna salobre (PC 4 al PC12) y a aquellos relacionados a la laguna cenagosa (PC 15 al PC 21) (Fig. 2). Sin embargo el análisis de clúster indica que la diferencia en composición de especies se acentúa entre los meses de mayo y julio, lo que puede ser explicado por la presencia de especies migratorias (Fig. 3). A partir de los meses de agosto en adelante se evidencia que la composición de especies en la laguna salobre y laguna cenagosa presenta una mayor similitud, pues se evidencia agrupamientos entre los PC de cada hábitat, sin embargo, entrando hacia el verano se evidencia un agrupamiento entre los PC 20 y 21 que corresponde a la zona de conexión de la laguna cenagosa con el mar.

Tabla 3. Lista de aves que hacen uso del humedal EI Paraiso.

\begin{tabular}{|c|c|c|c|c|c|c|c|}
\hline \multirow{2}{*}{$\mathbf{n}^{\circ}$} & \multirow{2}{*}{ Familia } & \multicolumn{2}{|c|}{ Estatus de conservación } & \multirow{2}{*}{$\mathbf{n}^{\circ}$} & \multirow{2}{*}{ Familia } & \multicolumn{2}{|c|}{ Estatus de conservación } \\
\hline & & $\begin{array}{c}\text { DS 04-2014 } \\
\text {-MINAGRI }\end{array}$ & IUCN & & & $\begin{array}{l}\text { DS 04-2014 } \\
\text {-MINAGRI }\end{array}$ & IUCN \\
\hline \multicolumn{2}{|r|}{ Anatidae } & & & 35 & Thalasseus maximus & & \\
\hline 1 & Anas bahamensis & & & 36 & Chroicocephalus cirrocephalus & & \\
\hline 2 & Anas cyanoptera & & & 37 & Leucophaeus atricilla & & \\
\hline 3 & Anas georgica & & & 38 & Larus belcheri & & \\
\hline 4 & Oxyurajamaicensis & & & 39 & Larus dominicanus & & \\
\hline \multicolumn{2}{|r|}{ Ardeidae } & & & 40 & Leucophaeus pipixcan & & \\
\hline 5 & Ardea alba & & & 41 & Leucophaeus modestus & & \\
\hline 6 & Ardea cocoi & & & \multicolumn{2}{|c|}{ Motacilidae } & & \\
\hline 7 & Bubulcus ibis & & & 42 & Anthus lutescens & & \\
\hline 8 & Butorides striata & & & \multicolumn{2}{|c|}{ Passeridae } & & \\
\hline 9 & Egretta caerulea & & & 43 & Passer domesticus & & \\
\hline 10 & Egretta thula & & & \multicolumn{2}{|c|}{ Pelecanidae } & & \\
\hline 11 & Egretta tricolor & & & 44 & Pelecanus thagus & EN & NT \\
\hline 12 & Ixobrychus exilis & & & \multicolumn{2}{|c|}{ Phalacrocoracidae } & & \\
\hline 13 & Nycticorax nycticorax & & & 45 & Phalacrocorax brasilianus & & \\
\hline \multicolumn{2}{|r|}{ Cathartidae } & & & \multicolumn{2}{|c|}{ Phoenicopteridae } & & \\
\hline 14 & Cathartes aura & & & 46 & Phoenicopterus chilensis & & NT \\
\hline 15 & Corapyps atratus & & & \multicolumn{2}{|c|}{ Podicipedidae } & & \\
\hline \multicolumn{2}{|c|}{ Charadriidae } & & & 47 & Podiceps major & & \\
\hline 16 & Charadrius alexandrinus & & & 48 & Podilymbus podiceps & & \\
\hline 17 & Charadrius vociferus & & & 49 & Rollandia rolland & & \\
\hline 18 & Pluvialis squatarola & & & \multicolumn{2}{|c|}{ Rallidae } & & \\
\hline \multicolumn{2}{|r|}{ Columbidae } & & & 50 & Fulica ardesiaca & & \\
\hline 19 & Columbia livia & & & 51 & Gallinula chloropus & & \\
\hline 20 & Zenaida meloda & & & 52 & Pardirallus sanguinolentus & & \\
\hline & Cuculidae & & & \multicolumn{2}{|c|}{ Recurvirostridae } & & \\
\hline 21 & Coccyzus melacoryphus & & & 53 & Himantopus mexicanus & & \\
\hline 22 & Crotophaga sulcirostris & & & \multicolumn{2}{|c|}{ Rynchopidae } & & \\
\hline \multicolumn{2}{|r|}{ Emberizidae } & & & 54 & Rynchops niger & & \\
\hline 23 & Sicalis luteola & & & & acidae & & \\
\hline & Falconidae & & & 55 & Actitis macularius & & \\
\hline 24 & Falco sparverius & & & 56 & Arenaria interpres & & \\
\hline & Furnariidae & & & 57 & Calidris $s p$. & & \\
\hline 25 & Geositta peruviana & & & 58 & Calidris alba & & \\
\hline 26 & Phleocryptes melanops & & & 59 & Calidris minutilla & & \\
\hline & Haematopodiadae & & & 60 & Limnodromus griseus & & \\
\hline 27 & Haematopus palliatus & & & 61 & Numenius phaeopus & & \\
\hline & Hirundinidae & & & 62 & Phalaropus tricolor & & \\
\hline 28 & Hirundo rustica & & & 63 & Tringa flavipes & & \\
\hline 29 & Pygochelidon cyanoleuca & & & 64 & Tringa melanoleuca & & \\
\hline & Icteridae & & & & kiornithidae & & \\
\hline 30 & Dives warszewiczi & & & 65 & Plegadis ridgwayi & & \\
\hline 31 & Sturnella bellicosa & & & & dytidae & & \\
\hline & Laridae & & & 66 & Troglodytes aedon & & \\
\hline 32 & Larosterna inca & & NT & & idae & & \\
\hline 33 & Sternula lorata & $\mathrm{EN}$ & $\mathrm{EN}$ & 67 & Pyrocephalus rubinus & & \\
\hline 34 & Thalasseus elegans & & NT & 68 & Tachuris rubrigastra & & \\
\hline
\end{tabular}


Si bien los hábitats acuáticos son los más representativos, en El Paraíso se evidenciaron patrones de agrupamiento entre los PC 1, 2 y 3, caracterizados por la presencia de carrizales, juncales, totorales y gramadales, y los PC 13 y 14, caracterizados por la presencia de totoral, juncal y salicornial (Fig. 2), donde predominan los ambientes terrestres. Es en ambas zonas donde se registraron el menor número especies e individuos de aves, que en su mayoría eran paseriformes.

El 31\% de las especies de aves se registraron únicamente en el hábitat de laguna salobre, el 34\% se registraron únicamente en el hábitat pantanoso, y el 35\% especies no presentaron preferencia por alguno de los hábitat evaluados.
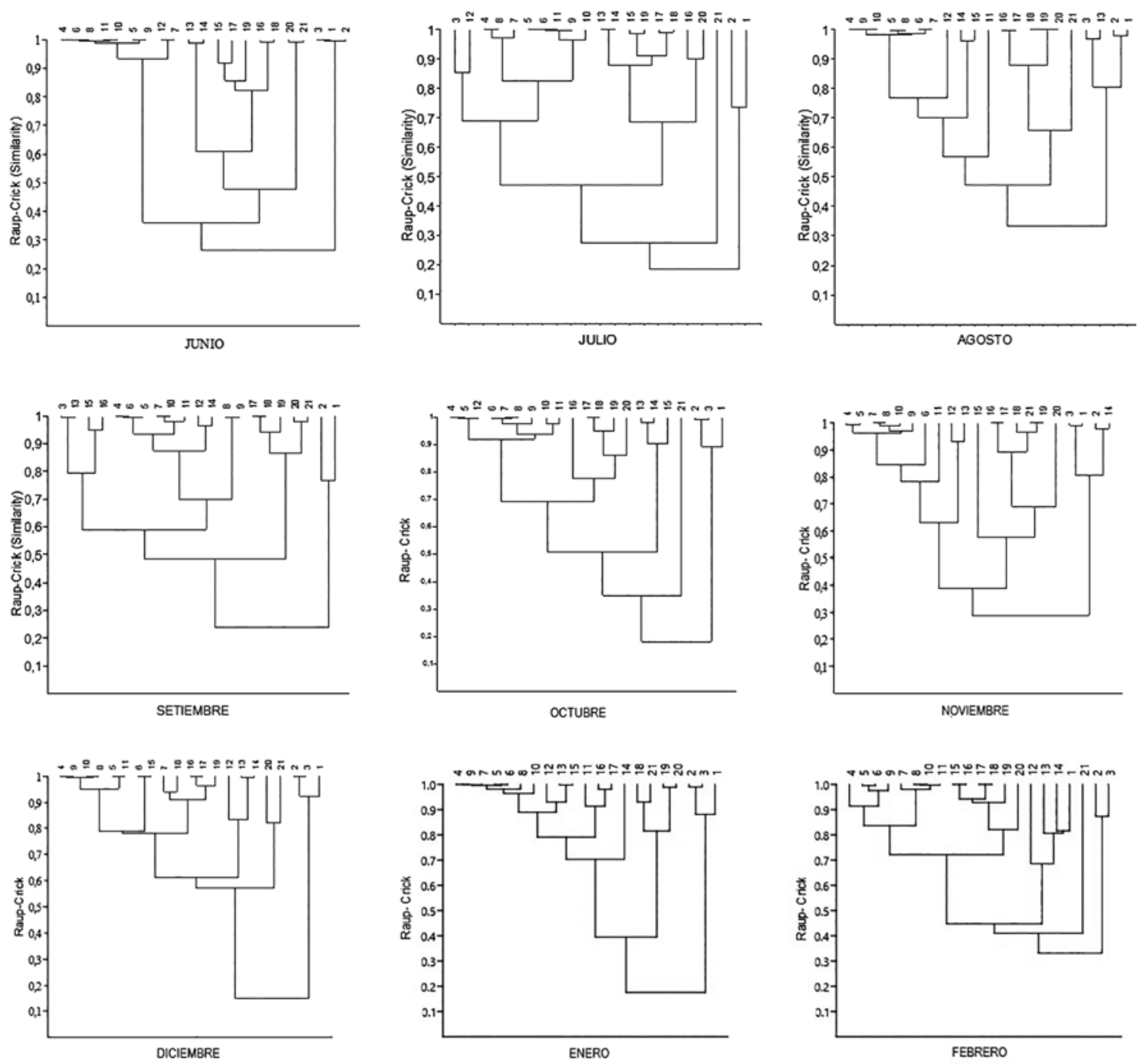

Figura 2. Análisis multivariado de Clúster, empleando el algoritmo de grupos pariados y el índice de similaridad de Raup-Crick para el periodo junio 2009 a febrero 2010.

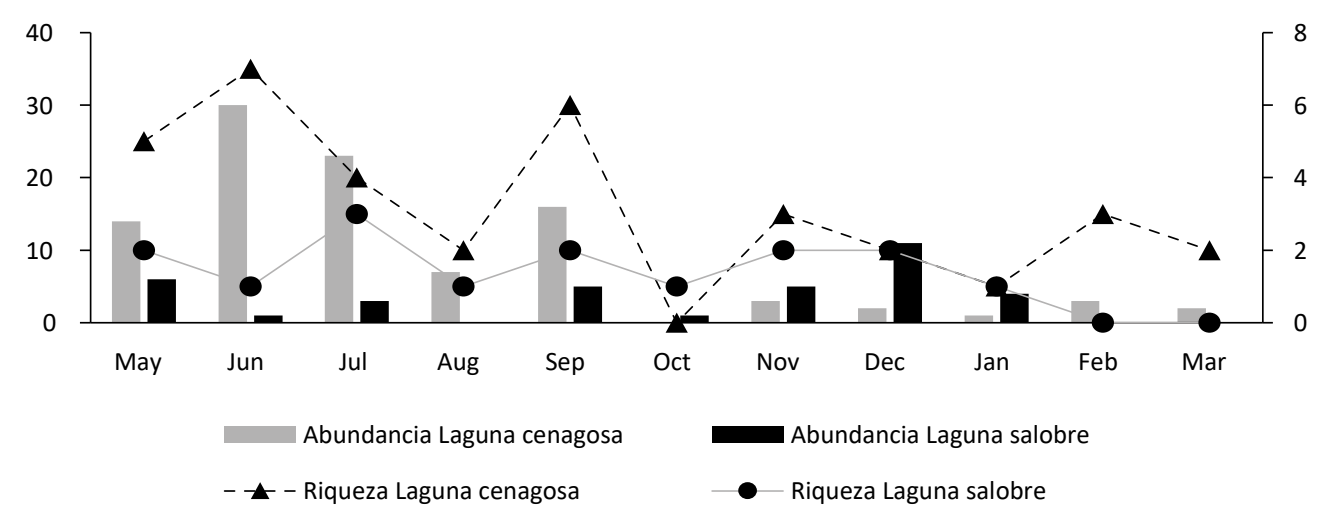

Figura 3. Variación temporal de la abundancia y riqueza de aves migratorias presentes en los dos hábitats evaluados durante el periodo de estudio, mayo 2009 a marzo 2010. 
Las diferencias en la composición de aves entre los dos hábitats se atribuyen, principalmente, a la presencia de especies migratorias boreales las cuales mostraron preferencia hacia el hábitat pantanoso (Tabla 4). Así mismo las especies Phoenicopterus chilensis, Plegadis ridgwayi y Haematopus paliatus presentaron una mayor abundancia y frecuencia de registro en el pantanal, sugiriendo una marcada preferencia por este hábitat, mientras que, Phalacrocorax brasilianus, Podiceps major y Oxyura jamaicensis presentaron mayor abundancia y frecuencia de registro en la laguna salobre (Fig. 4).

Esta preferencia de hábitat puede atribuirse a las características de nicho ecológico que presenta cada especie y a las características que cada uno de hábitats ofrece. Por ejemplo la laguna salobre presenta una mayor profundidad (Tabla 3), lo que permite a los Phalacrocoracidae y Podicipedidae bucear para conseguir su alimentos y desarrollar sus actividades diarias (Johnsgard 1993, Quintana et al. 2002, Fjeldså 2004), mientras que los Phoenicopteridae necesitan amplias zonas de niveles bajos de agua para conseguir su alimento mediante la filtración de zonas limosas de las lagunas (Tobar et al. 2014), al igual que los Charadriidae y Scolopacidae (Torres 2006).
Además, se observó preferencia de hábitat, claramente diferenciada, entre especies dentro de un mismo grupo taxonómico, como por ejemplo, Charadrius vociferus (Chorlo gritón) fue encontrado con mayor frecuencia en los alrededores de la laguna salobre, particularmente en los micro hábitats de gramadal y zonas limosas asociadas a juncales, mientras que Charadrius nivosus (Chorlo nevado) fue encontrado sólo en los alrededores de la zona pantanosa, en los microhábitat de limo y orilla marina. Aunque no pertenecen al mismo género las garzas también presentaron un patrón similar. Egretta tula (Garcita blanca) fue encontrada con mayor frecuencia en el pantanal mientras que Bulbucus ibis (Garcita bueyera) fue registrada mayormente en zonas terrestres y en la vegetación circundante a la laguna salobre.

Para el caso de los Chorlos las preferencias de hábitat no guardan relación con el tipo de actividad realizada. Sus hábitats de elección fueron utilizados tanto para actividades de alimentación como para descanso. Sin embargo en el caso de las garzas, la preferencia de hábitat se relacionó con su alimentación, ya que para el descanso se observó que ambas especies compartieron las zonas de juncal y totoral del humedal.

Tabla 4. Lista de especies de aves migratorias registradas durante el periodo de evaluación.

\begin{tabular}{|c|c|c|c|c|c|c|c|c|c|c|c|c|c|}
\hline Familia & Especie & $\begin{array}{l}\text { Estado de } \\
\text { migración }\end{array}$ & May & Jun & Jul & Aug & Sep & Oct & Nov & Dec & Jan & Feb & Mar \\
\hline Charadriidae & Charadrius alexandrinus & MB & & LC & $\mathrm{LC}$ & & LC & & LC & & & & \\
\hline \multirow[t]{5}{*}{ Laridae } & Larus atricilla & MB & $\mathrm{LC} / \mathrm{LS}$ & & & & & & & & & & \\
\hline & Leucophaeus modestus & MA & & & LS & & LS & & & & & & \\
\hline & Larus pipixcan & MB & LC & $\mathrm{LC}$ & & & $\mathrm{LC}$ & & & $\mathrm{LC}$ & LC/LS & $\mathrm{LC}$ & LC \\
\hline & Thalasseus elegans & MB & LC & & & & & & & & & & \\
\hline & Thalasseus maximus & MB & & $\mathrm{LC}$ & & & & & & & & & \\
\hline Recurvirostridae & Himantopus mexicanus & MB & LC & LC & LC & $\mathrm{LC}$ & LC & & LS & LS & & & \\
\hline \multirow[t]{8}{*}{ Scolopacidae } & Actitis macularia & MB & & & & & LS & LS & LS & LS & & & \\
\hline & Calidris $s p$ & MB & & & & & & & LC & & & & \\
\hline & Calidris minutilla & MB & & & & & & & & & & $\mathrm{LC}$ & \\
\hline & Limnodromus griseus & MB & & & & & & & $\mathrm{LC}$ & & & & \\
\hline & Numenius phaeopus & MB & & $\mathrm{LC}$ & LC & & $\mathrm{LC}$ & & & & & & \\
\hline & Phalaropus tricolor & MB & & & & & LC & & & & & & \\
\hline & Tringa flavipes & MB & LC & $\mathrm{LC}$ & & & LC & & & & & Lc & \\
\hline & Tringa melanoleuca & MB & & LC & LC & LC & & & & & & & \\
\hline Rynchopidae & Rhyncops niger & MA & LS & LS & LS & & & & & & & & \\
\hline Cuculidae & Coccyzus melacoryphus & MA & & & LS & LS & & & & & & & \\
\hline
\end{tabular}

LS = Laguna Salobre, $\mathrm{LC}=$ Laguna Cenagosa

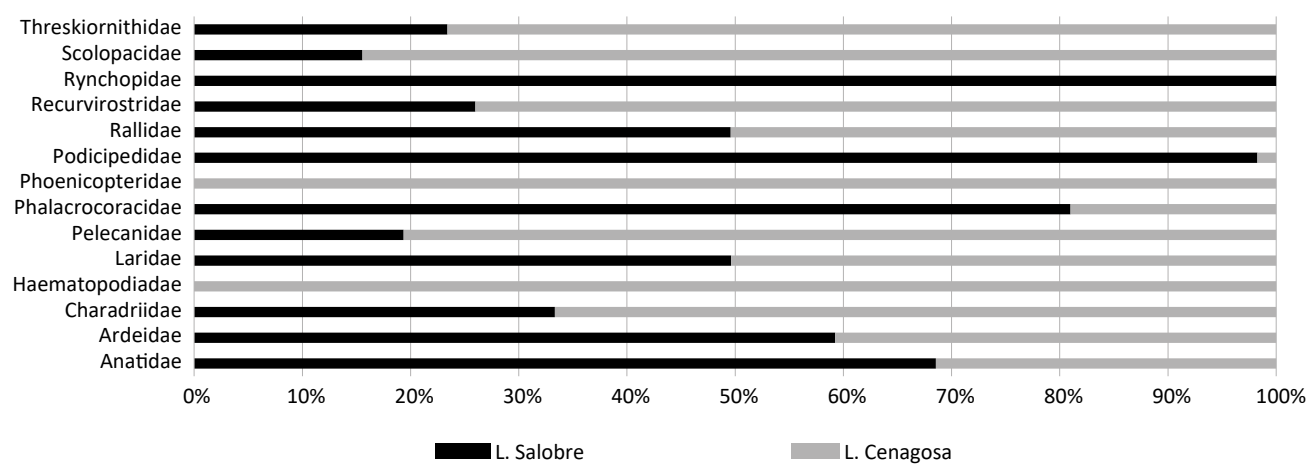

Figura 4. Frecuencia de avistamiento de individuos según familias de aves acuáticas. 
A diferencia de la mayoría de garzas, Bulbucus ibis se alimenta principalmente de insectos terrestres (Shulenberg et al. 2010) lo cual puede considerarse como una característica adaptativa, pues ha sido sugerido que las diferencias en el tipo de alimentación dentro de especies de un mismo grupo taxonómico, puede ser una estrategia para evitar competencia (Palacio-Núnez et al. 2008). Otro comportamiento considerado como una estrategia para evitar competencia es el de repartición temporal u horaria (Kronfeld-Schor \& Dayan 2003).

La principal actividad registrada para Nycticorax nycticorax durante el horario de evaluación, fue el descanso. Sin embargo, en la tarde-noche (fuera del horario de evaluación establecido) se evidencio mayor actividad de la especie, aunque no se le observó alimentándose. Palacios-Nuñez y colaboradoes (2008) sugieren que el hábito nocturno de $N$. nycticorax puede ser una estrategia de la especie para reducir su competencia con especies de aves diurnas.

Las aves de El Paraíso alternan sus periodos de alimentación y descanso en patrones no definidos y en circunstancias diversas.
La actividad frecuentemente registrada fue el descanso (73.9\%), seguido de la alimentación (23.4\%) y la reproducción (2.73\%). Se evidenció en la Laguna salobre una mayor frecuencia de registro para la actividad descanso y reproducción, mientras que la laguna pantanosa fue el hábitat donde se registró mayormente actividades de alimentación, este patrón fue similar a lo largo del periodo de evaluación (Fig. 5, Tabla 5).

Especies de interés.- El estudio permitió recopilar información sobre la historia natural y reproducción de algunas especies representativas del humedal. A continuación presentamos un resumen de la información colectada:

- Asio flameus: Especie rara en El Paraíso. Se registró a $A$. flameus en mayo 2009, forrajeando entre los juncales alrededor del canal de agua (PC1). Según Schulenberg y colaboradores (2010), A. flammeus es una especie rara, local en la costa y en pantanos. Sin embargo en evaluaciones previas, Tello y colaboradores (2008) registraron a la especie en diferentes ocasiones durante el verano

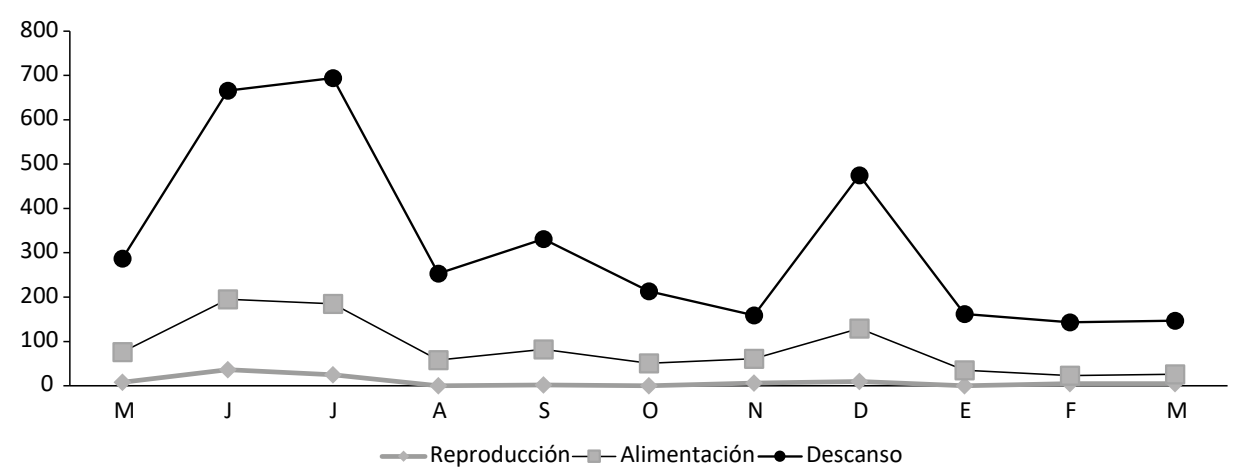

Figura 5. Variación temporal del comporamiento de la comunidad de aves de El Paraiso durante el periodo de estudio.

Tabla 5. Especies que presentaron alguna de las actividades de reproducción (cortejo, anidamiento) durante el periodo de estudio.

\begin{tabular}{lcc}
\hline \multicolumn{1}{c}{ Especie } & Laguna salobre & Laguna cenagosa \\
\hline Phalacrocorax brasilianus & $\mathrm{x}$ & \\
Podiceps major & $\mathrm{x}$ & \\
Podilymbus podiceps & $\mathrm{x}$ & $\mathrm{x}$ \\
Fulica ardesiaca & $\mathrm{x}$ & $\mathrm{x}$ \\
Gallinula chloropus & $\mathrm{x}$ & \\
Oxyura jamaicensis & $\mathrm{x}$ & \\
Nycticorax nycticorax & $\mathrm{x}$ & \\
Bubulcus ibis & $\mathrm{x}$ & $\mathrm{x}$ \\
Egretta thula & $\mathrm{x}$ & $\mathrm{x}$ \\
Anas cyanoptera & $\mathrm{x}$ & $\mathrm{x}$ \\
Anas bahamensis & & $\mathrm{x}$ \\
Leucophaeus modestus & $\mathrm{x}$ & \\
Charadrius nivosus & & \\
Geositta peruviana & $\mathrm{x}$ & \\
Pyrocephalus rubinus & $\mathrm{x}$ & $\mathrm{x}$ \\
Tachuris rubrigastra & $\mathrm{x}$ & $\mathrm{x}$ \\
Pheocryptes melanops & $\mathrm{x}$ & \\
\hline $\mathrm{x}$ observada en actividad reproductiva al & $\mathrm{x}$.nos una vez. & \\
\hline
\end{tabular}


del 2008 en El Paraíso. Así mismo Portocarrero (com. personal) la registró en la Albufera de Medio Mundo (32 km al norte de El Paraíso), en mayo del 2009. Estos registros confirman que $A$. flammeus es una especie que utiliza humedales costeros de Huaura-Lima como un área de refugio y alimentación.

- Geositta peruviana: Especie endémica, comúnmente encontrada al este del humedal, en zonas arenosas. Los registros corresponden, en su mayoría, a individuos solitarios. En noviembre 2009 se registró una pareja en cortejo, lo que puede indicar que $G$. peruviana puede estar reproduciéndose en el humedal o sus alrededores. A pesar de ser una especie común es poco lo que se conoce sobre su reproducción e historia natural (Shulenberg 2010, Salinas et al. 2007).

- Tachuris rubrigastra: Especie común en el humedal, relacionada a las zonas de juncales y totorales. Se evidenció actividad reproductiva (construcción de nidos, anidamiento y cuidado parental) entre octubre y diciembre 2009. Los nidos están hechos principalmente de juncos secos, formando una taza amarrada a una rama de totora. T. rubigastra es considerada un ave representativa ya que debido a sus variados y llamativos colores resulta atractiva para los observadores de aves.

- Phleocryptes melanops: Especie bastante común en el humedal. Se evidenció actividad reproductiva de la especie entre setiembre y diciembre 2009 entre los totorales adyacentes a la laguna salobre y en la vegetación (dominada por $S$. maritimus) alrededor de la zona pantanosa. Los nidos están hechos de pajas de totora y juncos, formando una taza amarrada a dos o más hojas de totora.

- Podiceps major: Es una de las especies más representativas dentro del humedal. Presenta marcada preferencia por la laguna salobre, pero al igual que a lo indicado por Tello $y$ colaboradores (2008) también ha sido registrado en grandes números en la zona de mar abierto en el humedal.

- Podiceps major presentó un ligero pico de reproducción entre mayo y julio 2009, sin embargo se observó actividad reproductiva (cortejo, anidamiento, cuidado parental) durante todo el año. Los cortejos consisten en danzas sincrónicas tal como lo descrito por Storer (1963). Se evidenció un fuerte cuidado parental y se registró un promedio de dos (02) crías por pareja. Esta especie nidifica en los totorales y juncales adyacentes a la laguna salobre, así como en las pequeñas islas de totora ubicadas al medio de esta laguna.

- Phalacrocorax brasilianus: Es una de las especies de mayor abundancia en el humedal. En abril 2009 se encontró una colonia (aprox. 60 individuos) realizando actividades reproductivas, anidamiento y cuidado parental. Los cormoranes hicieron sus nidos entre los juncales y totorales ubicados en el borde de la laguna salobre entre los PC 6 y 7 . El pico de reproducción registrado en este estudio fue de abril a junio 2009.

$\mathrm{Al}$ parecer este sería el primer registro, publicado, de $P$. brasilianus nidificando en un humedal costero del Perú. Las áreas de reproducción de $P$. brasilianus registradas a la fecha corresponden a islas y playas (Murphy 1936, Tovar 1968, Stucchi et al. 2011). Sobre su época de reproducción, tal y como se evidencia en este estudio, parece darse principalmente en el verano (Tovar 1968, Murphy 1936, Stucchi et al. 2011).

- Charadrius nivosus: Especie cuyas poblaciones del Pacífico, subespecie $C$. nivosus occidentalis, están protegidas bajo el acta de especies amenazadas (US Endangered Species Act, USFWS 1993). Es una especie común en el humedal, teniendo picos de abundancia en los meses de verano, donde se evidenció actividad reproductiva. Se encontraron cuatro (04) nidos en el mes de octubre y tres (03) nidos en noviembre, todos en el transecto cercano a la zona de orilla marina. Se evidenció actividad de crianza, hasta en febrero 2010.

Los nidos eran una pequeña cavidad circular en la arena revestido con pequeñas conchitas, contenían entre 2 a 3 huevos. Se evidenció un fuerte cuidado parental por parte de los padres, quienes vigilaban sigilosamente los nidos. Se georreferenció la ubicación de los nidos para su posterior monitoreo, pero todos fueron destruidos. Se encontraron marcas de llantas que pasaron sobre los puntos donde se encontraban los nidos destruidos. Küpper y colaboradores (2011) señalan que las actividades recreativas y económicas desarrolladas en playas arenosas, principales sitios de anidamiento de $C$. nivosus, son el más grave problema de conservación para la especie y en El Paraíso no es la excepción.

- Sternula lorata: Especie amenazada, categorizada como "En Peligro" según IUCN (BirdLife International, 2010). La presencia de S. lorata en el humedal fue común durante todo el periodo de evaluación. Se le registró en la zona de orilla marina, de mar abierto y pantano, lugares identificados como de descanso y alimentación. En setiembre 2009 se observó una pequeña colonia (8 individuos), entre los cuales se identificó a un juvenil, hacia la zona sur del humedal. Aunque este registro no es evidencia concreta de reproducción, estudios previos han identificado al humedal como un área de nidificación de la especie (Zavalaga et al. 2009).

- Phoenicopterus chilensis: Especie categorizada como "Casi amenazada” por IUCN (Birdlife 2011). P. chilensis es una especie común en el humedal. Presenta picos de abundancia en el verano donde se registraron algunos individuos con plumaje juvenil. P. chilensis presentó una marcada preferencia por el hábitat de pantano, que funciona como un área importante para el descanso y alimentación de la especie. Dentro de los humedales de la costa central del Perú, el Paraíso puede ser considerado un área estratégica para la conservación de $P$. chilensis puesto que es en este humedal donde se registran mayor abundancia de la especie y se le registra durante todo el año.

Conservación.- Los datos de uso de hábitat de las aves y las actividades antropogénicas nos permiten identificar las zonas de mayor vulnerabilidad dentro del humedal (Fig. 6).

La zona de limo y orilla marina, hacia el sur del humedal (PC $19,20,21)$ fue identificada como la más vulnerable, en especial 

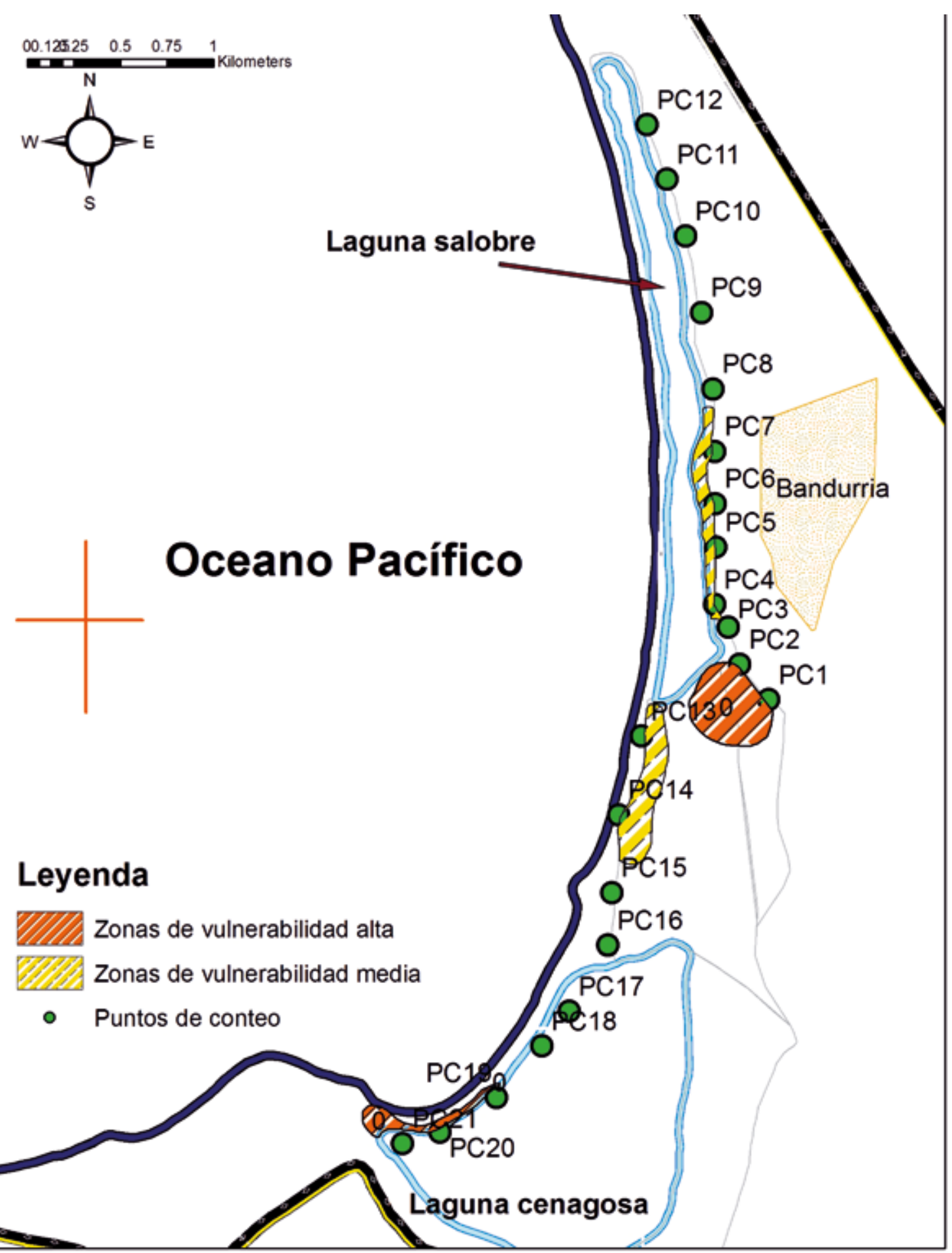

\section{Leyenda}

\section{Oceano Pacífico}

Zonas de vulnerabilidad alta

Zonas de vulnerabilidad media

- Puntos de conteo

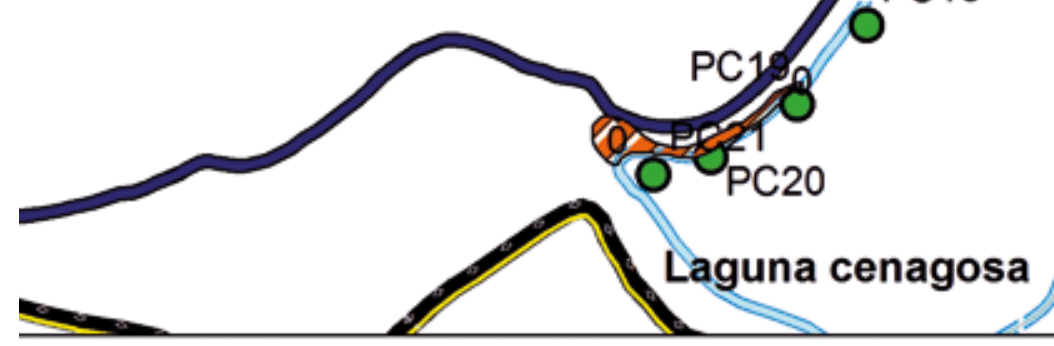

Figura 6. Las áreas delineadas en naranja son aquellas consideradas como zonas de vulnerabilidad alta, debido al registro de actividades de reproducción y actividades antropogénicas consideradas como amenazas directas a la conservación de aves. Las áreas delineadas en color amarillo son zonas de vulnerabilidad media, donde se han identificado zonas de reproducción de especies representativas del humedal tales como, Trachuris rubrigastra, Phleocryptes melanops, Phalacrocorax brasileanus.

durante el verano, debido a que es zona de reproducción de especies amenazadas como $S$. lorata y C. niviosus y a que se identificó el permanente tránsito de motos, camiones y camionetas, hay presencia de perros e inapropiado manejo de residuos sólidos dejados por los bañistas.

Otra de las zonas de importancia para la conservación de las aves en el Paraíso, es la zona de carrizales, totorales y jun- cales alrededor del canal de agua (PC 1 y 2). En esta área se ha identificado especies de importancia como A. flammeus, $C$. melacoryphus, y aves migratorias que aprovechan los parches limosos (p.e.: Calidris minutilla) para su alimentación. Además es un área importante para el descanso y alimentación de especies de hábitos terrestres como; Sturnella belicosa, Sicalis luteola, Pyrocephalus rubinus, entre otras (Fig. 6). 
Entre las principales amenazas observadas durante el periodo de evaluación tenemos; caza deportiva registradas en los meses de mayo y diciembre en la zona sur del humedal, quema, presencia de animales domésticos, introducción de especies exóticas, tránsito de vehículos, desecho de residuos sólidos, crecimiento urbano e inseguridad ciudadana.

Consideramos que cada uno de estos problemas debe de ser evaluado en detalle para establecer planes de manejo sostenible, prevención y búsqueda actividades económicas alternativas que sean amigables al ambiente y permitan la conservación de la avifauna.

El turismo de aves podría ser una actividad económica rentable dentro del humedal. El ecoturismo, de forma particular el turismo de observación de aves tiene una alta rentabilidad económica que a su vez favorece la conservación de los ecosistemas (PROMPERU 2014). Además esta actividad puede verse favorecida por que junto al humedal, en la terraza, se encuentra el centro Arqueológico Bandurria (Chu 2008).

\section{Agradecimientos}

Nuestro agradecimiento al Gobierno Regional de Lima en especial al ex Gerente de Recursos Naturales, ingeniero Luis Castillo Polo por las facilidades de materiales y equipos.. A Sheila Figueroa, Mónica Flores, Jesús Muñoz, Karen Verde, Luis Alza, Oscar Portocarrero, y demás estudiantes de la Universidad Nacional Agraria La Molina, Universidad Ricardo Palma y Universidad Peruana Cayetano Heredia por su colaboración con el trabajo de campo.

\section{Literatura citada}

Angulo F. 2009. Áreas Importantes para la Conservación de las Aves. Perú. Birdlife International: 307-316.

Bibby C., Burgess, N., Hill, D., \& S. Mustoe. 2000. Bird Census Techniques, 2nd ed. Academic Press, London. ISBN: 9780-12-095830-6

BirdLife International. 2016. Important Bird and Biodiversity Area factsheet: Laguna El Paraíso. Descargado de http://www. birdlife.org el 24/02/2016.

Blanco D. 1999. Los Humedales como Hábitat de aves acuáticas. Boletín UNESCO, Uruguay. : 208-217.

Brack A. 1986. Las ecorregiones del Perú. Bol. Lima 44: 57-70

Brandolin P. \& P. Blendinger. 2016. Effect of habitat and landscape structure on waterbird abundance in wetlands of central Argentina. Wetlands Ecology and Management 24(1):93-105. http://dx.doi.org/10.1007/s11273-015-9454-y

Castro, G. 1984. Las Aves de la Laguna El Paraíso. El volante Migratorio (3):10-11.

Castro G., Ortiz, E. \& L. Bertochi. 1990. Importancia Biológica y Conservación de la Laguna El Paraíso. Boletín de Lima (71):45-55

Chu A. 2008. Bandurria: Arena, Mar y Humedal en el Surgimiento de la Civilización Andina. Ediciones del Proyecto Arqueológico Bandurria, Huacho. Huacho. pp.185.

Chudzińska M., van Beest, F., Madsen, J. \& J. Nabe-Nielsen. 2015. Using habitat selection theories to predict the spatiotemporal distribution of migratory birds during stopover - a case study of pink-footed geeseAnser brachyrhynchus. Oikos 124(7):851-86. http://dx.doi.org/10.1111/oik.01881

Cruz Z., F. Angulo, H. Burger \& R. Borgesa. 2007. Evaluación de aves en la laguna El Paraiso, Lima, Perú. Revista Peruana de Biología 14(1):139-144 http://dx.doi.org/10.15381/ rpb.v14i1.2179

DS N ${ }^{\circ} 004-2015-M I N A N .2015$. Aprueban la Estrategia Nacional de Humedales. Sábado 24 de enero de 2015El Peruano Normal Legales: 545326 -Anexo.
Fjeldså J. 2004. The grebes, Podicipedidae. Oxford University Press, Oxford.

Hammer O., D.A.T. Harper, P.D. Ryan. 2001. PAST: Paleontological statistics software package for education and data analysis. Palaeontologia Electronica 4(1): 9pp. http://palaeo-electronica.org/2001_1/past/issue1_01.htm

Johnsgard, P. 1993. Cormorants, darters and 103 ABUNDANCIA DEL CORMORÁN CUELLO NEGRO pelicans of the world. Smithsonian Institution Press, Washington, DC.

Küpper C., E. Aguilar \& O. González. 2011. Notas sobre la ecología reproductiva y conservación de los chorlos nevados Charadrius nivosus occidentalis en Paracas. Revista Peruana de Biología 18(1): 091- 096. DOI: http://dx.doi.org/10.15381/ rpb.v18i1.153

Kronfeld-Schor N. \& T. Dayan. 2003. Partitioning of time as an ecological resource. Annual Review of Ecology, Evolution, and Systematics 34:153-81 http://dx.doi.org/10.1146/annurev. ecolsys.34.011802.132435

MINAM. 2011. Mapa de ecoregiones del Perú, escala 1/2000000. Mapa N-53.

Murphy R. 1936. Oceanic birds of South America. The Mac Millan Company. The American Museum of Natural History. New York, 2, $1245 \mathrm{p}$.

Myers J. 1983. Conservation of migrating shorebirds: Staging areas, geographic bottlenecks, and regional movements. American Birds 37:23-25

Núńez L. 1999. Guía de observación y monitoreo Aves de los $\mathrm{Hu}$ medales de Ventanilla. Alternativa, Centro de Investigación Social y Educación Popular. $108 \mathrm{p}$

Palacio-Núñez J., D. Jiménez-García, G. Olmos-Oropeza \& J. Enríquez-Fernández. 2008. Distribución y solapamiento espacial de las aves acuáticas y ribereñas en un humedal de zonas semiáridas del NE de México. Acta Zoológica Mexicana (n.s.) 24(2): 125-141.

PROMPERÚ. 2014. Perfil del Observador de aves 2013.

Quintana F., F. Morelli \& Y. Benedetti. 2002. Buceo eficiente en aguas poco profundas: comportamiento de buceo y patrón de alimentación del Cormorán Cuello Negro Phalacrocorax magellanicus, en dos colonias de la costa patagónica. Ecol. Austral 12: 19-28

Quiñonez A. \& A. Tello. 2011. Nuevos registros de Coccyzus melacoryphus en la costa del Perú. Journal Cotinga 33: 130-132.

Salinas L., C. Arana \& V. Pulido. 2007. Diversidad, abundancia y conservación de aves en un agroecosistema del desierto de Ica, Perú. Revista Peruana de Biología 13(3):155-168. http:// dx.doi.org/10.15381/rpb.v13i3.2333

Schulenberg T., D. Stotz, S. Lane, J. O’Neill \& T. Parker III. 2010. Aves del Perú. Princeton Univ. Press. Princeton, New Jersey.

Storer R. 1963. Observations on the Great Grebe. Condor 65:279-288.

Stucchi M. \& J. Figueroa. 2006. La avifauna de las islas Lobos de Afuera y algunos alcances sobre su biodiversidad. Reporte de investigación $N^{\circ} 2$. Asociación Ucumari. Lima, Perú. 88 pp. http://dx.doi.org/10.13140/RG.2.1.3608.0163

Tabilo E. 2003. El beneficio de los humedales en la Región Neotropical. Centro Neotropical de Entrenamiento en Humedales, La Serena - Chile: 73.

Tello A., A. Quińonez \& P. Merino. 2008. Evaluación de la avifauna local y migratoria en los Humedales de Paraíso. Gerencia Regional Recursos Naturales y Gestión de medio ambiente. Gobierno Regional de Lima.

Tello E., P. Sifuentes, J. Resurrección, J. Cabello, A. Sifuentes, J. Salvador \& A. Bustamante. 2014. Caracterización de sedimentos de la laguna El Paraiso, Huacho, Región Lima. Rev. Infinutum (4)1.

Tobar C.N., J.R. Rau, N. Fuentes, A. Gantz, C.G. Suazo, J.A. Cursach, A. Santibañez \& J. Pérez-Schultheiss. 2014. Diet of the Chilean flamingo Phoenicopterus chilensis (Phoenicopteriformes: Phoenicopteridae) in a coastal wetland in Chiloé, southern Chile. Revista Chilena de Historia Natural 87: 15. http://dx.doi.org/10.1186/s40693-014-0015-1.

Tovar H. 1968. Áreas de reproducción y distribución de las aves marinas en el litoral peruano. Boletín del Instituto del Mar del Perú, 1: 523-546 
Torres M., Z. Quinteros \& F. Takano. 2006. Variación temporal de la abundancia y diversidad de aves limícolas en el Refugio de Vida Silvestre Pantanos de Villa, Lima-Perú. Ecología Aplicada, 5: 119-125. http://dx.doi.org/10.21704/rea.v5i1-2.325

Weller M.W. 1999. Wetland Birds: Habitat Resources and Conservation Implications.--Milton W. Cambridge University Press, New York. $x v+277$ pp.
Riveros J., M. Cayo \& S. Núñez. 1983. Censos de Aves de Importancia Cinegética en las Albúferas de Playa Chica - Huacho. Zonas Áridas (3):75-81

Zavalaga C., J. Harvesty, G. Mori, C. Chávez-Villavicencio \& A. Te1lo. 2009. Current status of Peruvian Terns Sternula lorata in Perú: threats, conservation and research priorities. Bird Conservation International 19:175-186. 